\title{
Persistent Currents of Single Mesoscopic Rings.
}

\author{
U. ECKERN $\left(^{*}\right)$ and A. SCHMID $\left({ }^{* *}\right)$ \\ ${ }^{*}$ ) Kernforschungszentrum Karlsruhe, Institut für Nukleare Festkörperphysik \\ Postfach 3640, W-7500 Karlsruhe 1 \\ ${ }^{(* *)}$ Institut für Theorie der Kondensierten Materie, Universität Karlsruhe \\ Postfach 6980, W-7500 Karlsruhe 1
}

PACS. 73.20F - Weak localization effects.

PACS. 05.30F - Fermion systems and electron gas.

PACS. 75.20E - Metals and alloys.

\begin{abstract}
We study the stochastic fluctuations (from sample to sample) in the persistent current $I(\phi)$ of mesoscopic rings threaded by a flux $\phi$. We find that it is necessary to include the Coulomb interaction between the electrons. Then we obtain $\left\langle I^{2}\right\rangle^{1 / 2} \sim(L / l)\langle I\rangle \sim e v_{\mathrm{F}} / L$; and $h / e$ for the dominant period in $\phi$. This agrees well with a recent experiment on single gold rings. Furthermore, we predict that the persistent currents have a Gaussian probability distribution, since the total electron number of a typical ring is still very large.
\end{abstract}

Most recently, a group at IBM [1] has measured the flux dependence of the persistent current $I(\phi)$ in single mesoscopic gold rings. They found that the dominant period of $I(\phi)$ is $\phi_{0}=h / e$; moreover, and most surprisingly, they observed a current of the order $e v_{F} / L(L$ is the perimeter of the ring) which is about two orders of magnitude larger than what has been found by a group at Bell Labs. [2] for the average current $\langle I(\phi)\rangle$ of an ensemble comprising $10^{7}$ copper rings. If one does not wish to believe in large differences between the two materials, gold and copper, one has to assume large stochastic fluctuations from sample to sample, even if they have been fabricated identically on a mesoscopic level. In this letter, we report on our investigations of such stochastic fluctuations in the persistent current.

Our theory follows the concept set out by Ambegaokar and Eckern [3] where the Coulomb interaction between the electrons has been found to be responsible for the observed [2] magnitude of the average persistent current $\langle I(\phi)\rangle$. There it was found that $\langle I\rangle \sim T_{1} / \phi_{0}$, where $T_{1}=\hbar D / L^{2}$ is the Thouless energy $\left(D=v_{\mathrm{F}} l / 3\right.$ is the diffusion constant, and $l=v_{\mathrm{F}} \tau$ the elastic mean free path).

The ansatz of ref. [3] is based on selected Hartree-Fock-type contributions to the grand canonical potential, as considered earlier in various contexts [4]. In particular, arguments were brought forth on a logarithmic renormalization of the coupling constant (cf. [4]); but these we believe, are not appropriate for the stochastic fluctuations considered here. For simplicity, we proceed on the assumption that the simple Hartree diagram is sufficient; and we will show that from this assumption, it follows $\left\langle I^{2}\right\rangle^{1 / 2} /\langle I\rangle \sim\left(\tau T_{1} / \hbar\right)^{-1 / 2} \sim$ $\sim L / l \gg 1$. 
Taking averages of products with respect to the random impurity positions, we find it convenient to define connected correlators. Of central importance will be the connected two-point correlation function

$$
\mathscr{M}_{I}\left(\phi, \phi^{\prime}\right)=\left\langle I(\phi) I\left(\phi^{\prime}\right)\right\rangle_{\mathrm{con}}=\left\langle I(\phi) I\left(\phi^{\prime}\right)\right\rangle-\langle I(\phi)\rangle\left\langle I\left(\phi^{\prime}\right)\right\rangle .
$$

It is found to be of the form

$$
\mathscr{M}_{I}\left(\phi, \phi^{\prime}\right)=\mathscr{F}\left(\phi-\phi^{\prime}\right)-\mathscr{F}\left(\phi+\phi^{\prime}\right),
$$

where the two terms on the right-hand side of this equation arise from the diffuson and cooperon contribution, respectively. Note that $\mathscr{F}$ is an even function; it has the representation

$$
\mathscr{F}(\phi)=\sum_{m=1}^{\infty} f_{m} \cos \left(2 \pi m \phi / \phi_{0}\right),
$$

where for zero temperature, $T=0$, and zero phase breaking rate, $\gamma=0$,

$$
f_{m}(T=0, \gamma=0)=\frac{32}{m} \frac{a}{a+2} \frac{T_{1} \hbar / \tau}{\phi_{0}^{2}}=\frac{8 \alpha}{3 \pi^{2}(\alpha+2)} \frac{1}{m}\left(\frac{e v_{F}}{L}\right)^{2} .
$$

Here $a=\kappa^{2} / 2 k_{\mathrm{F}}^{2} \sim 1$, where $\kappa$ denotes the Thomas-Fermi screening wave vector. The quantity $f_{m}(T, \gamma) / f_{m}(0,0)$ is given by the same expression as found for the $(T, \gamma)$-dependence of the average current [3]; hence for $y=0$, it decreases with increasing temperature approximately according to $\exp \left[-m^{2} T / 3 T_{1}\right]$, while for $T=0$, finite phase breaking leads to

$$
f_{m}(0, \gamma) / f_{m}(0,0)=\left(1+m \Gamma_{1}^{1 / 2}\right) \exp \left[-m \Gamma_{1}^{1 / 2}\right] ; \quad \Gamma_{1}=\hbar \gamma / T_{1} .
$$

We briefly outline the theoretical basis and the derivation of the above results. Since the persistent current is an equilibrium quantity, we need to study properties of the grand potential $\Omega(\phi)$ and, in particular, the connected correlation function

$$
\mathscr{M}_{\Omega}\left(\phi, \phi^{\prime}\right)=\left\langle\Omega(\phi) \Omega\left(\phi^{\prime}\right)\right\rangle_{\mathrm{con}}
$$

from which $\mathscr{M}_{I}$ is determined via differentiation with respect to $\phi$ and $\phi^{\prime}$. As mentioned, we consider the Hartree contribution to $\Omega$ only; in addition, for the screened Coulomb interaction, we use the Thomas-Fermi expression $\tilde{u}(k)=4 \pi e^{2} /\left(k^{2}+\kappa^{2}\right)$.

In computing the impurity average in (6), we have to connect $\Omega(\phi)$ and $\Omega\left(\phi^{\prime}\right)$ by impurity lines, e.g., as shown in fig. $1 a$ ); and in order to determine the flux dependence, we have to extract the dominant pole contributions of the diffuson and of the cooperon (the particle-hole and the particle-particle impurity ladders). In view of the complexity of the analytical expression, we propose two methods for its evaluation.

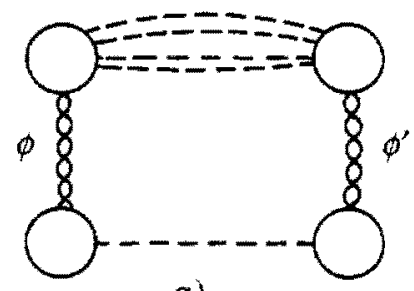

a)

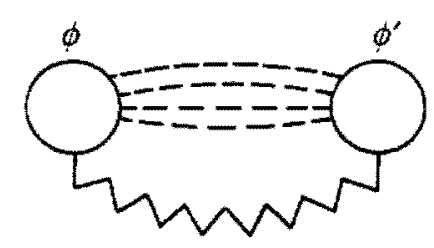

b)

Fig. 1. - a) Graphical representation of $\left\langle\Omega(\phi) \Omega\left(\phi^{\prime}\right)\right\rangle_{\mathrm{con}}$ within the Hartree approximation. Double wavy line: screened Coulomb interaction. Dashed lines arise from impurity averaging. $b$ ) Equivalent representation for $a$ ), in which the lower part has been condensed into a pseudointeraction (zig-zag line). 
I) Since the momentum going around the loop in fig. 1a) is large and of order $|\boldsymbol{k}| \sim k_{\mathrm{F}}$, we expect the dominant flux dependence to be given by the term with a single diffuson/cooperon, as shown in this figure. Then we recognize that formally (see fig. 1b)) we may introduce a pseudo interaction $W(k)$, given by (note the combinatorial factor of four)

$$
W(\boldsymbol{k})=4 \cdot 1 / 2 \cdot\left[2 \mathscr{N}^{0} \tilde{u}(\boldsymbol{k})\right]^{2} \cdot \hbar / 2 \pi \mathscr{N}^{0} \tau .
$$

From the analogy with the calculation [3] of the average thermodynamic potential, we easily deduce the following result:

$$
\mathscr{M}_{\Omega}\left(\phi, \phi^{\prime}\right)=2 \mathscr{N}^{0} \bar{W} T \sum_{\omega, q}\left(\frac{|\omega|}{|\omega|+\gamma+D q^{2}}\right)_{\mathrm{d}+c},
$$

where presently we have to sum the diffuson (d) and cooperon (c) contributions. Note that for the ring situation (transverse dimension much smaller than the perimeter), the wave vector $q$ has to be taken one-dimensional such that

$$
\boldsymbol{q} \rightarrow q_{\mathrm{c}, \mathrm{d}}=\frac{2 \pi}{L}\left(n-\frac{\phi \pm \phi^{\prime}}{\phi_{0}}\right)
$$

with $n$ an integer, and $\Sigma_{q} \rightarrow \Sigma_{n}$. Inserting the Fermi surface average $\bar{W}$ (defined as earlier in ref. [3]) of the pseudo interaction $W$, we arrive at the results (2)-(4). We remark that the large typical value of $|\boldsymbol{k}| \sim k_{\mathrm{F}}$ renders the coupling constant renormalization [4] due to multiple interactions in the Cooper channel ineffective, in contrast to the calculation of the average current [3].

II) As an alternative, we write $\mathscr{M}_{\Omega}$ in the form (')

$$
\mathscr{M}_{\Omega}\left(\phi, \phi^{\prime}\right)=2 \cdot \frac{\mathscr{V}}{4} \int \frac{\mathrm{d}^{3} k}{(2 \pi)^{3}}[\tilde{u}(\boldsymbol{k})]^{2} \cdot\left[\mathcal{Q}\left(\boldsymbol{k} ; \phi, \phi^{\prime}\right)\right]^{2},
$$

where the diffuson contribution to $\mathcal{Q}\left(k ; \phi, \phi^{\prime}\right)$ is shown graphically in fig. 2 . Clearly, there is a dominant "regular" contribution, $\mathscr{Q}_{\mathrm{r}}$, arising from the region $\left|\boldsymbol{k}_{1}-\boldsymbol{k}_{2}\right|>l^{-1}$, which is given by (a single impurity line suffices for its evaluation) $\mathcal{Q}_{\mathrm{r}}=2 \hbar \mathscr{N}^{0} / \pi \tau$. On the other hand, a small but flux sensitive («singular») contribution, $\mathcal{2}_{\mathrm{s}}$, arises from the region $\left|k_{1}-k_{2}\right|<l^{-1},\left|\varepsilon_{1}-\varepsilon_{2}\right|<\hbar / \tau$, which for the ring case in order of magnitude $\left(|k| \sim k_{\mathrm{F}}\right)$ is given by $\mathcal{Q}_{\mathrm{s}} \sim\left(T_{1} / \varepsilon_{\mathrm{F}}\right) \mathscr{V}^{-1}$, where $\varepsilon_{\mathrm{F}}$ is the Fermi energy. Hence $\mathcal{Q}_{\mathrm{s}} / \mathcal{Q}_{\mathrm{r}} \sim(l / L)^{2} / N$ is extremely small $\left(N \sim \mathcal{\nu} k_{F}^{3} \sim \mathcal{Q}^{\prime} N^{0} \varepsilon_{F}\right.$ is the number of electrons), and it is sufficient to retain

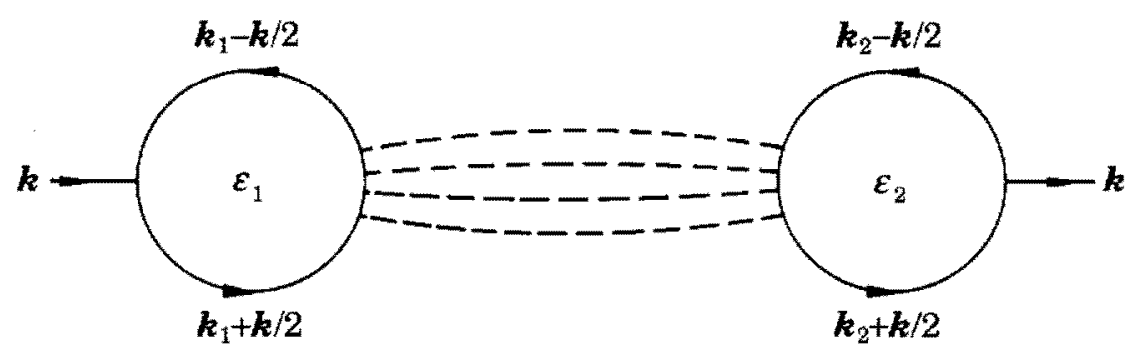

Fig. 2. - Representation of the diffuson contribution to $\mathcal{Q}\left(k ; \phi, \phi^{\prime}\right)$, as explained in connection with eq. (9).

(1) Note the multiplicity factor of two. $\mathscr{Q}$ is the volume of the system. 


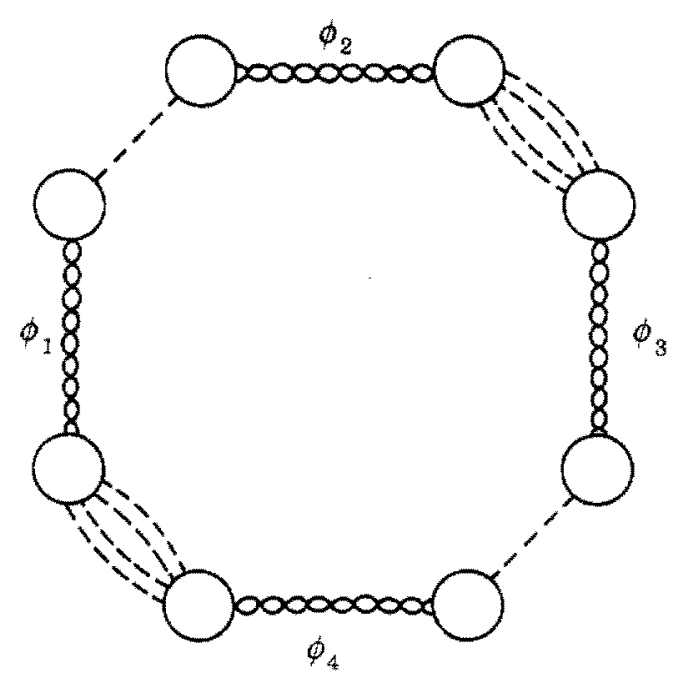

Fig. 3. - Contribution to the connected 4-point correlation function.

only the mixed term $\sim \mathcal{Q}_{\mathrm{r}} \mathcal{Q}_{\mathrm{s}}$ in (10). Proceeding to evaluate $\mathscr{M}_{\Omega}$ along this method, we restrict $|\boldsymbol{k}|$ to the range $0 \ldots 2 k_{\mathrm{F}}$, and confirm the result (8).

The analysis along method II) suggests the following estimates of higher-correlation functions. Define $\mathscr{M}_{\Omega}^{(n)}$ to be the connected $n$-point correlation function, i.e.

$$
\mathscr{M}_{\Omega}^{(n)}\left(\phi_{1}, \ldots, \phi_{n}\right)=\left\langle\Omega\left(\phi_{1}\right), \ldots, \Omega\left(\phi_{n}\right)\right\rangle_{\mathrm{con}},
$$

it is clear that this quantity has a dominant regular part (compare fig. 3), which is given by $\sim N(\hbar / \tau)^{n}$. However, the quantity of interest is the connected current correlation function, which is obtained from (11) by differentiating with respect to all variables $\phi_{1}, \ldots, \phi_{n}$ (and multiplying with $(-1)^{n}$ ); hence we have to determine, out of all contributions to (11), only those which are sensitive to all fluxes $\phi_{1}, \ldots, \phi_{n}$. As is apparent from fig. 3 and since $\mathcal{Q}_{\mathrm{r}} \gg \mathcal{Q}_{\mathrm{s}}$, the dominant term is the one where we retain the singular (impurity ladder) contribution for every second impurity connection between $\Omega$ 's ( $n$ even). Suppressing the flux dependence for simplicity, we find for

$$
n=2 p: \quad \mathscr{M}_{\Omega}^{(2 p)} \sim N \cdot\left(\mathscr{N}^{0}\right)^{-n} \mathcal{Q}_{\mathrm{r}}^{p} \mathcal{Q}_{\mathrm{s}}^{p}
$$

and for

$$
n=2 p+1: \quad \mathscr{M}_{\Omega}^{(2 p+1)}-N \cdot\left(\mathscr{N}^{0}\right)^{-n} \mathcal{Q}_{\mathrm{r}}^{p} \mathcal{Q}_{\mathrm{s}}^{p+1}
$$

Inserting the estimates of $\mathcal{Q}_{\mathrm{r}}, \mathcal{Q}_{\mathrm{s}}$ as given above, the estimate for the connected current correlation functions is (compare (4) where $p=1$ )

$$
\begin{gathered}
\mathscr{M}_{I}^{(2 p)} \sim\left(e v_{\mathrm{F}} / L\right)^{2 p} N^{1-p} \\
\mathscr{M}_{I}^{(2 p+1)} \sim(l / L)\left(e v_{\mathrm{F}} / L\right)^{2 p+1} N^{-p}
\end{gathered}
$$

Hence in units of $\left(e v_{\mathrm{F}} / L\right)^{n}$ we obtain $\mathscr{M}_{I}^{(n)} \sim N^{1-n / 2}$ and $\mathscr{M}_{I}^{(n)} \sim(l / L) N^{(1-n) / 2}$ for $n$ even and odd, respectively. At that point we remind that the number $N$ of electrons is large even for mesoscopic samples $\left(N \sim 10^{10}\right)$. Therefore, only the two-point correlation function is 
important and the higher-order correlators can be neglected. As a consequence, the probability distribution of the persistent currents is Gaussian $\left({ }^{2}\right)$.

Recall eqs. (2) and (3) and note that $\mathscr{M}_{I}\left(\phi, \phi^{\prime}\right)$ is an odd function of $\phi$ and $\phi^{\prime}$. Consequently, the even eigenfunctions of the operator $\mathscr{M}_{I}$ have eigenvalue zero; and it follows that $I(\phi)$ is odd with probability one. Therefore,

$$
I(\phi)=\sum_{m=1}^{\infty} I_{m} \sin \left(2 \pi m \phi / \phi_{0}\right)
$$

where the Fourier coefficients $I_{m}$ are independent Gaussian variables with probability distribution

$$
P\left(\left\{I_{m}\right\}\right) \sim \exp \left[-\sum_{m=1}^{\infty} \frac{\left(I_{m}-\left\langle I_{m}\right\rangle\right)^{2}}{2 \sigma_{m}^{2}}\right] .
$$

For orientation note that at $T=\gamma=0$, one finds $\sigma_{m}^{2} \equiv 2 f_{m} \sim\left(e v_{\mathrm{F}} / L\right)^{2} / m$; recall also $\left\langle I_{2 k+1}\right\rangle=$ $=0$, and that $\left\langle I_{2 k}\right\rangle \sim\left(e v_{\mathrm{F}} l / L^{2}\right) k^{-2} \ll \sigma_{2 k}$ is negligeably small. We emphasize that the current is an odd function of the flux (as it should be) only if the cooperon and diffuson contribute with the same amplitude.

At this point, we wish to emphasize an important difference between the stochastic fluctuations of persistent currents and the Aharonov-Bohm effect in mesoscopic normal-metal rings [5-7]. In the latter case, one may expand [8] the measured quantity (i.e. the conductance) in a Fourier series $\propto \cos \left[2 \pi m \phi / \phi_{0}+\gamma_{m}\right]$, in a way similar to eq. (16). However, the phases $\gamma_{m}$ for different values of $m$ appear to be completely uncorrelated with no theoretical prediction available. Therefore, only the power spectrum (with respect to $\phi$ ) of the measured quantity is physically relevant; in fact, this is the central message of the so-called ergodic hypothesis [7]. In contrast to that, the phase of the persistent current is uniquely fixed by the condition that the current be an odd function of $\phi$.

In summary, we find that the persistent current $I(\phi)$ of one mesoscopic ring is an odd function of the flux $\phi$ with Fourier components $I_{m} \sin \left(2 \pi m \phi / \phi_{0}\right)$. The amplitude $I_{m}$ is a stochastic function depending on the random position of the impurities with a mean value $\left\langle I_{m}\right\rangle$ almost zero. Using data of ref. [1], we find

$$
\left\langle I_{1}^{2}\right\rangle^{1 / 2}=\sigma_{1} \sim 0.42 e v_{\mathrm{F}} / L .
$$

This value, and the period $h / e$, are in good agreement with the experimental results obtained for three gold rings [1]. We expect the current to vary randomly in sign from ring to ring; and from one Fourier component to the other. (Experimentally, the sign of the current for two rings was found to be positive, but obviously no conclusion can be drawn from such a limited sampling.) Detailed data [1] are given for a $(1.4 \times 2.6) \mu \mathrm{m}^{2}$ loop $(L=8 \mu \mathrm{m})$; for this loop, we compute the characteristic temperature $3 T_{1} \sim 12 \mathrm{mK}$. Thus for $T \sim 7.6 \mathrm{mK}$, the second harmonic is suppressed by a factor $\sim 13$, consistent with its almost absence in the power spectrum ([1], fig. 2(d)). Concerning the parity of the current and its derivatives, we feel that fig. $2 b$ and $2 c$ of ref. [1] are of fair illustration of $I(-\phi)=-I(\phi)$.

Finally, in order to develop an idea of the width of the dominant $h / e$-peak in the power spectrum, we consider a flux-dependent phase-breaking rate $\gamma(\phi)$, as for example arises from the finite transverse dimension $(\sim 900 \AA)$ of the loop. For the quoted parameters, we

(2) For large $p$, small momenta $|k|-l^{-1}$ are found to be important, which leads to factors $\sim\left(k_{\mathrm{F}} l\right)^{p-3}$ and $\sim\left(k_{\mathrm{F}} l\right)^{p-2}$ for $p>3$ and $p>2$, in (14) and (15), respectively. These, however, do not change the conclusion. 
find $\left(^{3}\right)$ [4] $\gamma_{\mathrm{H}}=1 / \tau_{\mathrm{H}}=\beta^{2}\left(\phi / \phi_{0}\right)^{2} T_{1} / \hbar$ with $\beta \approx 0.5$. Hence, we estimate the relative width ( (full width at half maximum") as $\beta / \pi \sim 0.16$ (experimentally it is about twice this value), indicating that field penetration gives a substantial contribution to the broadening. Clearly, more detailed studies of the lineshape will be most interesting.

We thank Y. IMRY and V. AMBEGAOKAR for interesting comments and suggestions. This work is part of a project within the SFB 195 «Lokalisierung von Elektronen in makroskopischen und mikroskopischen Systemen».

(3) See ref. [9] for a discussion of various phase breaking effects in isolated metal rings.

\section{REFERENCES}

[1] Chandrasekhar V., Webb R. A., Brady M. J., Ketchen M. B., Gallagher W. J. and Kleinsasser A., Phys. Rev. Lett., 67 (1991) 3578.

[2] Levy L. P., Dolan G., Dunsmuir J. and Bouchiat H., Phys. Rev. Lett., 64 (1990) 2074; Levy L. P., Physica B, 169 (1991) 245.

[3] AMBEgaOKAR V. and ECKern U., Phys. Rev. Lett., 65 (1990) 381; 67 (1991) 3192.

[4] Altshuler B. L., Aronov A. G., Khmelnitskir D. E. and Larkin A. L., in Quantum Theory of solids, edited by I. M. LIFshitz (MIR Publishers, Moscow) 1980, p. 130; ALTshuler B. L. and Aronov A. G., in Electron-Electron Interactions in Disordered Systems, edited by A. L. EFros and M. Pollak (North-Holland, Amsterdam) 1985, p. 1.

[5] Webb R. A., Washburn S., Umbach C. P. and Laibowitz R. B., Phys. Rev. Lett., 54 (1985) 2696; Washburn S. and WebB R. A., Adv. Phys, 35 (1986) 375.

[6] Altshuler B. L., Pis'ma Zh. Eksp. Teor. Fiz, 41 (1985) 530 (JETP Lett, 41 (1985) 648).

[7] Lee P. A. and Stone A. D., Phys. Rev. Lett., 55 (1985) 1622.

[8] Stone A. D. and IMry Y., Phys. Rev. Lett., 56 (1986) 189.

[9] Eckern U., Z. Phys. B, 82 (1991) 393. 